\title{
Transcriptional upregulation of c-MET is associated with invasion and tumor budding in colorectal cancer
}

\author{
Conor A. Bradley, ${ }^{1,}$, Philip D. Dunne ${ }^{1, *}$, Victoria Bingham $^{1}$, Stephen McQuaid ${ }^{1,2}$, \\ Hajrah Khawaja ${ }^{1}$, Stephanie Craig ${ }^{1}$, Jackie James ${ }^{1,2}$, Wendy L. Moore ${ }^{1}$, Darragh \\ G. McArt ${ }^{1}$, Mark Lawler ${ }^{1}$, Sonali Dasgupta ${ }^{1}$, Patrick G. Johnston ${ }^{1}$, Sandra Van \\ Schaeybroeck ${ }^{1}$ \\ ${ }^{1}$ Drug Resistance Group, Centre for Cancer Research and Cell Biology, School of Medicine, Dentistry and Biomedical Science, \\ Queen's University Belfast, Belfast, UK \\ ${ }^{2}$ Tissue Pathology, Belfast Health and Social Care Trust, Belfast City Hospital, Belfast, UK \\ *Joint first authors
}

Correspondence to: Sandra Van Schaeybroeck, email: s.vanschaeybroeck@qub.ac.uk

Keywords: $C-M E T$, colorectal cancer (CRC), tumor budding, invasion, metastasis

Received: July 06, 2016 Accepted: October 19, $2016 \quad$ Published: October 26, 2016

\section{ABSTRACT}

C-MET and its ligand HGF are frequently overexpressed in colorectal cancer (CRC) and increased C-MET levels are found in CRC liver metastases. This study investigated the role of the HGF/C-MET axis in regulating migration/invasion in CRC, using preclinical models and clinical samples. Pre-clinically, we found marked upregulation of C-MET at both protein and mRNA levels in several invasive CRC cells. Down-regulation of C-MET using RNAi suppressed migration/invasion of parental and invasive CRC cells. Stimulation of CRC cells with rh-HGF or co-culture with HGF-expressing colonic myofibroblasts, resulted in significant increases in their migratory/invasive capacity. Importantly, HGF-induced c-MET activation promoted rapid downregulation of c-MET protein levels, while the MET transcript remained unaltered. Using RNA in situ hybridization (RNA ISH), we further showed that MET MRNA, but not protein levels, were significantly upregulated in tumor budding foci at the invasive front of a cohort of stage III CRC tumors $(p<0.001)$. Taken together, we show for the first time that transcriptional upregulation of MET is a key molecular event associated with CRC invasion and tumor budding. This data also indicates that RNA ISH, but not immunohistochemistry, provides a robust methodology to assess MET levels as a potential driving force of CRC tumor invasion and metastasis.

\section{INTRODUCTION}

Colorectal cancer (CRC) is a major cause of cancer related death worldwide, with significant variation in prognosis between early and late stage disease. Five year overall survival (OS) rates for patients with localised stage II disease are as high as $80 \%$ following surgery, dropping to $40-60 \%$ for patients with stage III disease who display evidence of tumor invasion and regional lymph node metastasis [1]. Tumor budding is defined by the presence of individual tumor cells and/or small clusters of cancer cells at the invasive front of tumors [2]. Tumor budding at the invasive front has long been associated with presence of lymph node and distant metastasis, increased risk of relapse and is now a well-accepted prognostic factor in
CRC [3-5]. However, the underlying biology driving the distinct prognostic differences between localised tumors and those which have developed an increased invasive capacity is, as yet, not fully understood at the molecular level.

Recent studies have highlighted the considerable intra-tumoral heterogeneity that exists within CRC primary tumors. A recent consensus molecular classification of stage II/III CRC has identified the poor prognostic CMS 4 subgroup, characterized by epithelial-mesenchymal transition (EMT) and stem-like transcriptional signatures [6]. Further interrogation of this CRC subgroup revealed the stromal-rich nature of these tumors, particularly the presence of fibroblasts, which account for the high levels of mesenchymal associated genes expressed within the 
CMS 4 subtype [7, 8]. These studies indicated that the prognostic value of the CMS 4 subtype is inherently associated with fibroblast infiltration, and suggested that widespread EMT does not occur within the epithelial tumor bulk. Other studies have shown that the interaction between epithelial cancer cells at the invasive front or budding cells with surrounding stromal cells or their secreted factors can locally affect Wnt/ $\beta$-catenin signalling within these cells, triggering stemness, EMT and an invasive behaviour, suggesting that this pathway may be an important driver of the metastatic process and potential therapeutic target in budding cells [9-12].

The interplay between cancer associated fibroblasts (CAFs) and tumor cells, leading to metabolic reprogramming of cancer and stromal cells and activation of an EMT and invasive programme, has been described. Tumor-derived secreted factors such as TGF- $\beta 1$, bFGF, and IL- 6 , have been shown to control the activation of cancer associated fibroblasts (CAFs), and the proteome/ secretome of these CAFs, including hepatocyte growth factor (HGF), has been found to enhance the invasiveness of cancer cells $[13,14]$. The prognostic and predictive value of tumor-infiltrating fibroblasts is not a new concept [15-17]. The recent tumor profiling efforts $[18,19]$ have also failed to identify specific epithelial-derived factors associated with the extremely small proportion of cells undergoing invasion and budding at the invasive front. In order to model CRC cell invasion/metastasis, our group has developed invasive CRC daughter cells, which display stem-like characteristics, an EMT phenotype and increased migratory/invasive levels compared to their parental counterparts $[20,21]$.

The receptor tyrosine kinase c-MET (mesenchymalepithelial transition factor), encoded by the MET protooncogene, and its cognate high-affinity ligand HGF control invasive growth through the coordination of cell proliferation, survival, EMT and migration/invasion [22, 23]. c-MET expression in CRC primary tumors has been found to be predictive of local tumor invasion and regional lymph node metastasis [24] and higher c-MET levels have been found in synchronous CRC liver metastasis compared to levels obtained in matched primary tumors $[25,26]$. We have previously shown that MEK1/2 inhibition-induced c-MET activation is an acute mechanism of resistance to MEK1/2 inhibitors in RAS and BRAF mutant CRC [27, 28].

In this study, we show that c-MET protein and mRNA levels are significantly increased in our CRC invasive models and that treatment with c-MET-specific siRNA abrogates migration/invasion of parental and invasive CRC cells. We also demonstrate that incubation of CRC cells with rh-HGF or co-culture with HGFexpressing myofibroblasts significantly increases migration/invasion and this was associated with rapid downregulation of c-MET protein but not mRNA levels. Using RNA in situ hybridization (RNA ISH) and immunohistochemistry (IHC), we show high MET mRNA but not protein levels in tumor budding foci at the invasive front of stage III CRC tumors. Taken together, our results indicate for the first time that elevated MET mRNA levels is associated with increased invasive capacity in vitro and the presence of tumor budding in vivo, suggesting that c-MET targeted therapies may represent a promising strategy to prevent invasion and disease recurrence in stage III CRC.

\section{RESULTS}

\section{c-MET is a key regulator of CRC cell invasion and migration in vitro}

In order to identify molecular drivers of invasion and metastasis, we generated preclinical in vitro invasive HCT116 and HKH-2 CRC models which display an EMT-like invasive phenotype [20,21]. Initially, we also developed invasive cell populations from the LoVo CRC cell line, using Matrigel Invasion Chambers. Using the XCELLigence real-time cell migration/invasion tracking system, we confirmed the invasion/migration rates of our invasive models, and found a 35.7-fold, 3.29-fold and 27.7-fold increase in migration rates $(P<0.001)$ and 6.65-fold, 1.64-fold and 128-fold increase in invasion rates $(P<0.001)$ in invasive HCT116, HKH-2 and LoVo cells respectively (Figure 1A). We assessed MET gene expression in these invasive models using qRT-PCR and found marked upregulated MET mRNA levels in the invasive subpopulations compared to their parental counterparts (Figure 1B). Increased MET mRNA levels were associated with increased c-MET protein levels in invasive subpopulations as compared to their matched parental cells (Figure 1C). Interestingly, we also observed marked increases in c-MET phosphorylation levels at kinase domain residues $\mathrm{Y}^{1234 / 1235}$ in all our invasive cell models, indicating that increased kinase activity of the receptor was concordant with c-MET overexpression (Figure 1C). Quantification of human HGF (h-HGF) protein secretion by ELISA illustrated that both parental and invasive CRC cell lines did not secrete physiologically detectable levels of h-HGF into the culture medium, indicating that overexpression and phosphorylation of c-MET in invasive cell lines is independent of autocrineHGF secretion (Figure 1D). Consistent with this finding, we observed no detectable $H G F$ mRNA levels in our CRC cell line models (Supplementary Figure S1).

In order to investigate a potential role for c-MET in regulating migration and invasion, we employed different siRNA sequences directed against MET in the HCT116, HKH-2, LoVo, and DLD-1 parental and invasive CRC cells. Using the XCELLigence real-time cell migration tracking system, we found that loss of MET gene expression resulted in statistically significant attenuation of relative migration rate in parental and invasive cell line 
models, with a 44-92\% reduction compared to scrambled control (SC)-treated cells $(p<0.001$ for all cell line models) (Figure 2A and Supplementary Figure S2A). This effect was also evident using in vitro Boyden chamber assays, where siMET resulted in marked reduced invasion in HCT116 and DLD-1 cell lines (Figure 2B). Importantly, the decreased migration/invasion rates observed following siMET was not due to increased cell death or changes in the cell cycle profile (Supplementary Figure S2B).

\section{HGF-mediated activation of c-MET promotes CRC cell migration and invasion}

We assessed the role of exogenous $\mathrm{HGF}$ in promoting migration and invasion of CRC cells. In order to model the CRC tumor microenvironment (TME), we used the normal colonic myofibroblast cell line CCD$18 \mathrm{Co}$ to represent tumor-associated stromal fibroblasts. Initial phenotypic characterisation showed that HCT116 cells displayed a change from an epithelial to an elongated, spindle-shaped mesenchymal morphology $24 \mathrm{~h}$ following incubation with conditioned medium derived from CCD18Co cells (CCD-18CoCM) (Supplementary Figure S3A). Using an indirect co-culture system, we observed marked increased migration and invasion of HCT116 and LoVo cells following $48 \mathrm{~h}$ co-culture with CCD-18Co cells (Figure 3A). Although incubation of CRC cells with CCD-18CoCM for $72 \mathrm{~h}$ resulted in a 1.2 -fold and 1.5 -fold increased proliferation rates in HCT116 and LoVo cells respectively, the increased invasion rates of HCT116 and LoVo cells when co-cultured with CCD-18Co cells occurred already within the first $24 \mathrm{~h}$ (Supplementary Figure S3B).

In order to identify the signalling mechanism which drives increased migration/invasion in our CRC models following co-culture with CCD-18Co cells, we assessed the phosphorylation status of 42 RTKs using a human phospho-RTK array kit. The phospho-RTK array images and matched densitometry data showed significant increased c-MET tyrosine phosphorylation levels in HCT116 (4.67-fold; $p<0.001)$ and LoVo (91.39fold; $p<0.01)$ cells following 15-minutes incubation with CCD-18CoCM compared to the unstimulated cells (Figure 3B). We validated our array results by Western blotting, using anti- $\mathrm{Y}^{1234 / 1235} \mathrm{c}-\mathrm{MET}$ and total c-MET antibodies. Phosphorylation of $\mathrm{Y}^{1234 / 1235} \mathrm{c}-\mathrm{MET}$, but not total c-MET levels, was markedly increased in HCT116 and LoVo cells following 15 min incubation with CCD18CoCM (Figure 3B). Further data showed significant increases in $H G F$ mRNA and protein levels in CCD-
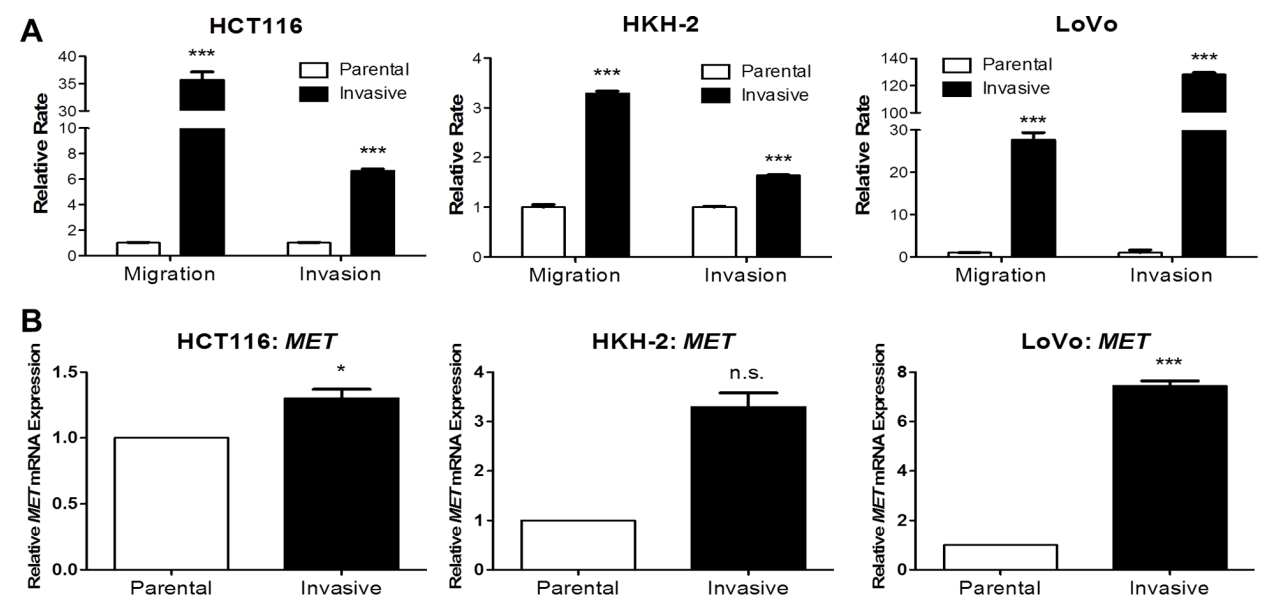

C
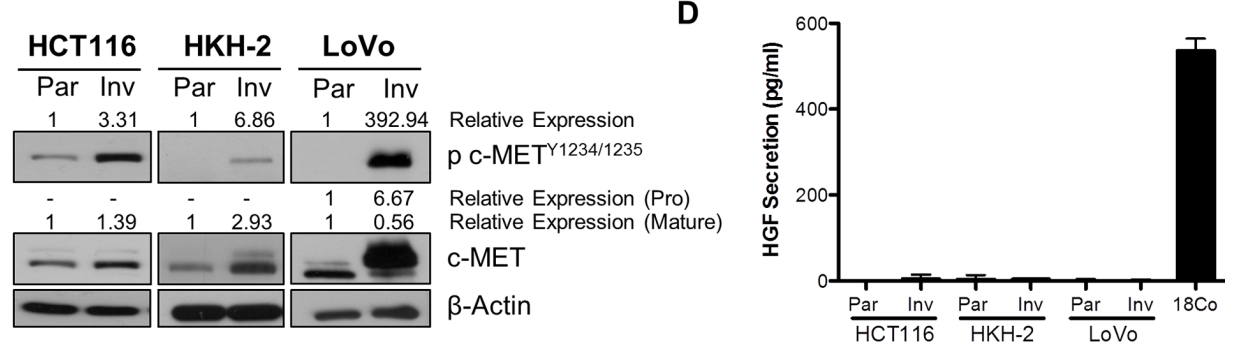

Figure 1: c-MET protein and mRNA levels are highly upregulated in invasive CRC daughter cell lines. BD BioCoat Matrigel invasion chambers were used to isolate invasive subpopulations from a panel of CRC cells. (A) Migration and invasion assays of invasive subpopulations of HCT116, HKH-2 and LoVo sublines were compared with the parental cells using the quantitative xCELLigence system. (B) qRT-PCR assessment of MET gene expression levels in parental and invasive cell models. (C) Western blot analysis of pcMET $^{1234 / 1235}$ and c-MET protein expression levels in parental (Par) and invasive (Inv) cell models. (D) HGF protein levels in the culture media of parental (Par) and invasive (Inv) subpopulations were measured by ELISA [Positive control: CCD-18Co $(18 \mathrm{Co})]$. $*=p<0.05$, $* * *=p<0.001$, n.s. $=$ not significant. 
18Co cells compared to levels measured in the HCT116 cells (Supplementary Figures S1 and S3C), indicating that myofibroblast-derived $\mathrm{HGF}$ plays a key role in c-MET activation and migration/invasion of CRC cells following co-culture with CCD-18Co cells. In addition, stimulation of HCT116 and LoVo cells with recombinant human HGF (rh-HGF) $(25 \mathrm{ng} / \mathrm{ml})$ resulted in significant increases in migration $(p<0.001)$ and invasion $(p<$ 0.001 ) rates (Figure 4A), confirming the key role of the $\mathrm{HGF} / \mathrm{c}-\mathrm{MET}$ axis in mediating CRC cell migration and invasion in the context of the TME. siRNA-mediated knockdown of MET and neutralisation of HGF using an anti-HGF monoclonal antibody was sufficient to abrogate the increased migratory/invasive potential of CRC cells following co-culture with CCD-18Co cells (Figure 4B and 4C).

\section{Recombinant and myofibroblast-derived HGF promotes dynamic downregulation of c-MET protein levels}

In order to assess the effect of HGF stimulation on the stability of c-MET protein levels, we performed a time-course experiments with rh-HGF treatment in our CRC cells. Marked increases in c-MET ${ }^{\mathrm{Y} 1234 / 1235}$ levels were observed in HCT116 and LoVo cell lines, $15 \mathrm{~min}$ and 1 hour following stimulation with $25 \mathrm{ng} / \mathrm{ml} \mathrm{rh}-\mathrm{HGF}$; however at the latter time-point, downregulation of total c-MET levels was observed (Figure 5A). Similar results were seen following co-culture of HCT116 and LoVo cells with CCD-18Co fibroblasts (Figure 5B). In contrast to c-MET protein levels, MET mRNA levels remained unchanged, 6, 12 or 24 hours following stimulation with rh-HGF (Figure 5C). These data highlight that the unstable nature of c-MET protein levels is not reflected at the transcriptional level, and therefore that $M E T$ mRNA expression levels may be a more robust readout of biological overexpression and dependency on c-MET.

\section{MET transcription, but not protein expression, is upregulated in budding CRC cell populations in stage III primary CRC tumors}

To support the results of our in vitro experiments, we examined MET gene and protein expression in situ in budding tumor cells at the invasive front using CRC clinical tissues. Budding tumor cells at the CRC invasive front are potentially exposed to high physiological concentrations of stromal-derived paracrine ligands through either direct or indirect contact. Using whole face sections of primary stage III CRC tumors (Table 1, $\mathrm{n}=13$ ), we performed RNA in situ hybridisation (RNA ISH) using a probe specific for MET (Figure 6A). While there appeared to be only small fluctuations in transcription levels across the entire section, we performed detailed examination of multiple regions at the invasive front compared to the central tumor. Using a digital pathology method (See Materials and Methods) to measure the signal intensity, converting signal intensity into a digital surrogate measure of single cell transcript levels, we found a significant increase in $M E T$ transcription in budding cells compared to the central tumor regions $(p<0.001)$ (Figure 6A). While there was some variation in the baseline transcript levels in the central regions between tumors, we consistently observed an increase in the transcript levels in budding cells across all 13 tumor budding-positive tumors examined, regardless of the associated central region transcriptional levels within each
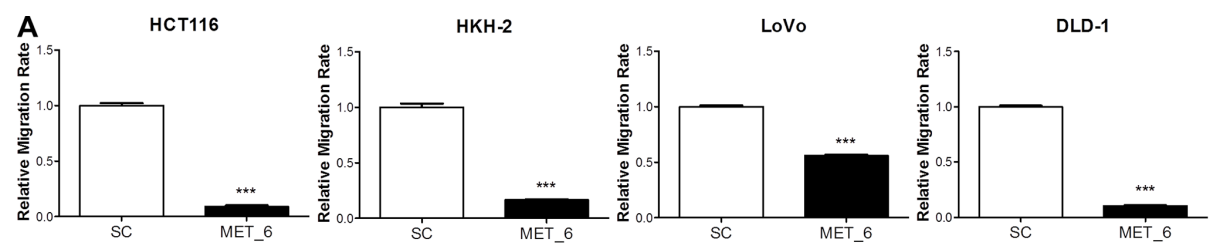

SC MET_6 siRNA/10nM

SC MET_6 siRNA/10nM

SC MET_6 siRNA/10nM

SC MET_6 siRNA/10nM
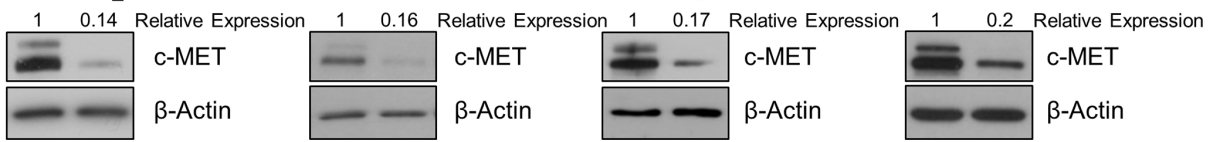

B
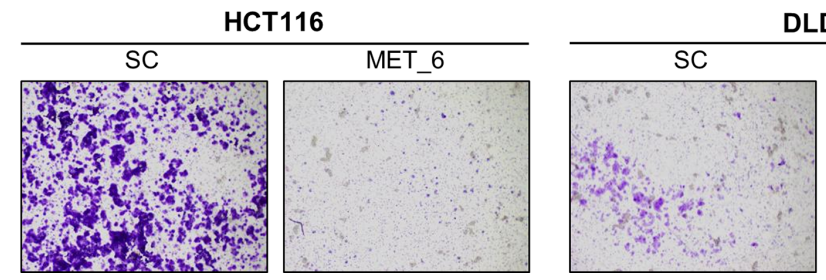

DLD-1

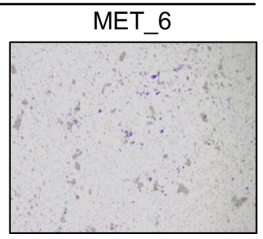

Figure 2: c-METi reduces migration and invasion of CRC cell lines. (A) CRC cells were transfected with $10 \mathrm{nM} \mathrm{SC}$ or $10 \mathrm{nM}$ c-MET siRNA (MET_6) for 24 hours and the effect on migration was determined using the xCELLigence system. Corresponding c-MET protein knockdown was confirmed by Western blotting. (B) Qualitative invasion rate assessment was performed following transfection with $10 \mathrm{nM}$ SC or 10nM c-MET siRNA (MET_6), using Boyden chambers. ${ }^{* * *}=p<0.001$. 
tumor $(p<0.001)$ (Figure 6B). The cell-specific source of $M E T$ expression was confirmed by both molecular analysis and pathology-based assessment. Using Haematoxylin and Eosin (H\&E) evaluation of the whole tissues sections and E-Cadherin staining, MET gene expression was localised to the epithelial cells (Supplementary Figure S4). In addition, bioinformatics assessment of microarray profiles obtained from dissociated fresh primary tumors (GSE39396), which had been fluorescence activated cell sorting (FACS) selected into specific endothelial, epithelial, leukocyte and fibroblast populations, confirmed that MET gene expression is significantly higher in tumor epithelial cells when compared to endothelial cells or leukocyte or fibroblast cells within the TME $(p<0.0001)$ (Supplementary Figure S5).

As our in vitro models showed instability of c-MET protein following co-culture with CCD-18Co cells and stimulation with rh-HGF, we assessed c-MET protein levels in our clinical tissues. IHC analysis revealed that although differences in basal c-MET protein levels could be detected between individual tumors, no increased c-MET protein levels were observed between the central and budding cells in individual tumors (Supplementary Figure S6).

\section{DISCUSSION}

The establishment of metastasis following the development of a primary tumor, requires a number of well-defined steps, however the molecular factors associated with metastatic progression still remain poorly understood [29]. Our previous data and other studies have identified a role for AXL in cancer cell migration/invasion and showed that AXL levels are required for niche activation and the first phase of metastatic colonization $[21,30]$. However, delineation of the key molecular drivers of the initial invasive event in CRC tissues requires a detailed investigation of single budding cells at the invasive front, which will open the way for novel and tailored approaches to target metastatic spread and increase outcome of patients with early stage CRC.

Using unsupervised classification of gene expression profiles, a number of research groups have published

\section{A}
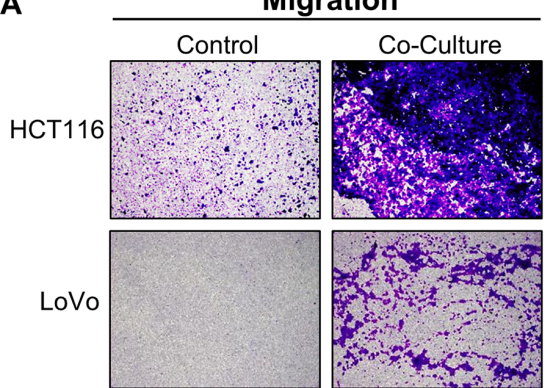

B

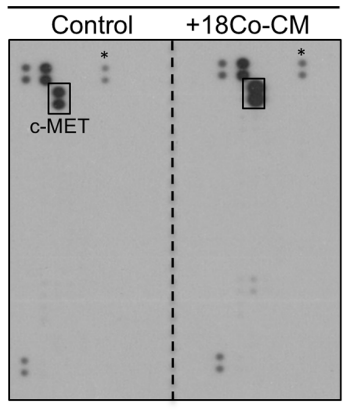

HCT116: c-MET

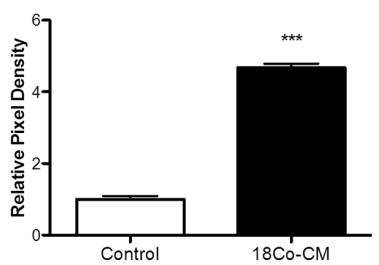

Invasion
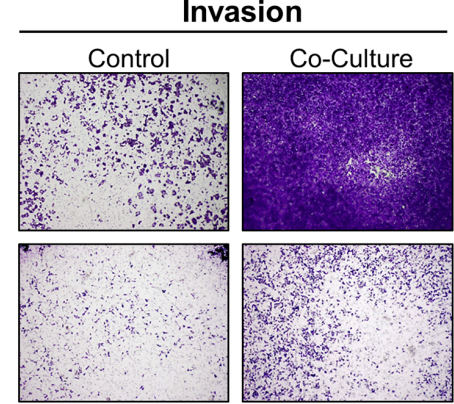

LoVo

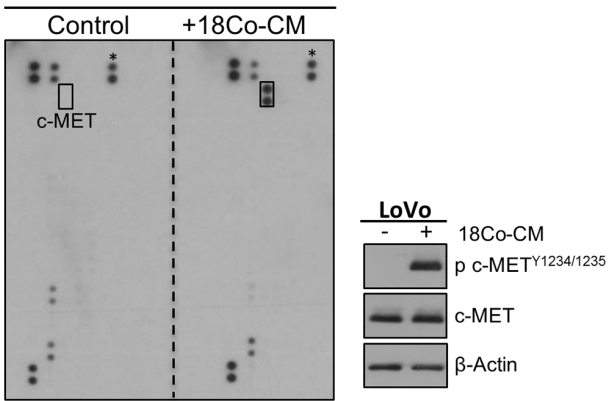

LoVo: c-MET

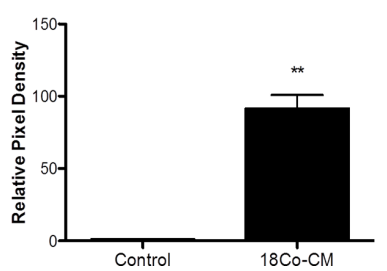

Figure 3: Myofibroblast-derived HGF promotes migration and invasion of CRC cells. (A) HCT116 and LoVo cell lines were indirectly co-cultured with the colon fibroblast cell line CCD-18Co and the effect on both migration and invasion was determined using Boyden chambers. (B) Human phospho-receptor tyrosine kinase (pRTK) array in HCT116 and LoVo cells, cultured with or without conditioned media from CCD-18Co fibroblast cell line (18Co-CM). Total phosphorylated MET protein levels was further quantified by pixel density assessment and Western blotting. $*=$ Reference for normalisation. 
Table 1: Clinico-pathological features of the stage III CRC patient cohort

\begin{tabular}{|c|c|}
\hline Patient characteristics & $N=13$ \\
\hline \multicolumn{2}{|l|}{ Age (y) } \\
\hline Median & 64 \\
\hline Range & $39-73$ \\
\hline \multicolumn{2}{|l|}{ Gender } \\
\hline Male & $8(62 \%)$ \\
\hline Female & $5(38 \%)$ \\
\hline \multicolumn{2}{|l|}{ T Stage } \\
\hline pT0 & 0 \\
\hline pT1 & 0 \\
\hline pT2 & 0 \\
\hline pT3 & $6(46 \%)$ \\
\hline pT4a & 0 \\
\hline pT4b & $7(54 \%)$ \\
\hline \multicolumn{2}{|l|}{ N Stage } \\
\hline N0 & 0 \\
\hline N1 & $9(69 \%)$ \\
\hline $\mathrm{N} 2$ & $4(31 \%)$ \\
\hline \multicolumn{2}{|l|}{ M Stage } \\
\hline M0 & $13(100 \%)$ \\
\hline M1 & 0 \\
\hline \multicolumn{2}{|l|}{ Staging } \\
\hline IIB & 0 \\
\hline IIIA & $1(8 \%)$ \\
\hline IIIB & $8(61 \%)$ \\
\hline IIIC & $4(31 \%)$ \\
\hline IV & 0 \\
\hline \multicolumn{2}{|l|}{ Tumor Type } \\
\hline Adenocarcinoma & $12(92 \%)$ \\
\hline Mucinous Carcinoma & $1(8 \%)$ \\
\hline \multicolumn{2}{|l|}{ Differentiation } \\
\hline Well - Moderate & $11(77 \%)$ \\
\hline Poorly & $3(23 \%)$ \\
\hline \multicolumn{2}{|l|}{ Tumor Site } \\
\hline Caecum & $5(39 \%)$ \\
\hline Ascending Colon & $2(15 \%)$ \\
\hline Transverse Colon & 0 \\
\hline Sigmoid Colon & $3(23 \%)$ \\
\hline Splenic Flexure & $1(8 \%)$ \\
\hline Rectum & $2(15 \%)$ \\
\hline \multicolumn{2}{|l|}{ Lymphovascular Invasion (LVI) } \\
\hline Yes & $10(76 \%)$ \\
\hline No & $3(24 \%)$ \\
\hline
\end{tabular}


Extramural Venous Invasion (EMVI)

Yes

$4(31 \%)$

No

$9(69 \%)$

Perineural Invasion (PI)

Yes

0

No

Uncertain

Tumor Budding

Yes

$13(100 \%)$

No

0

A

Migration
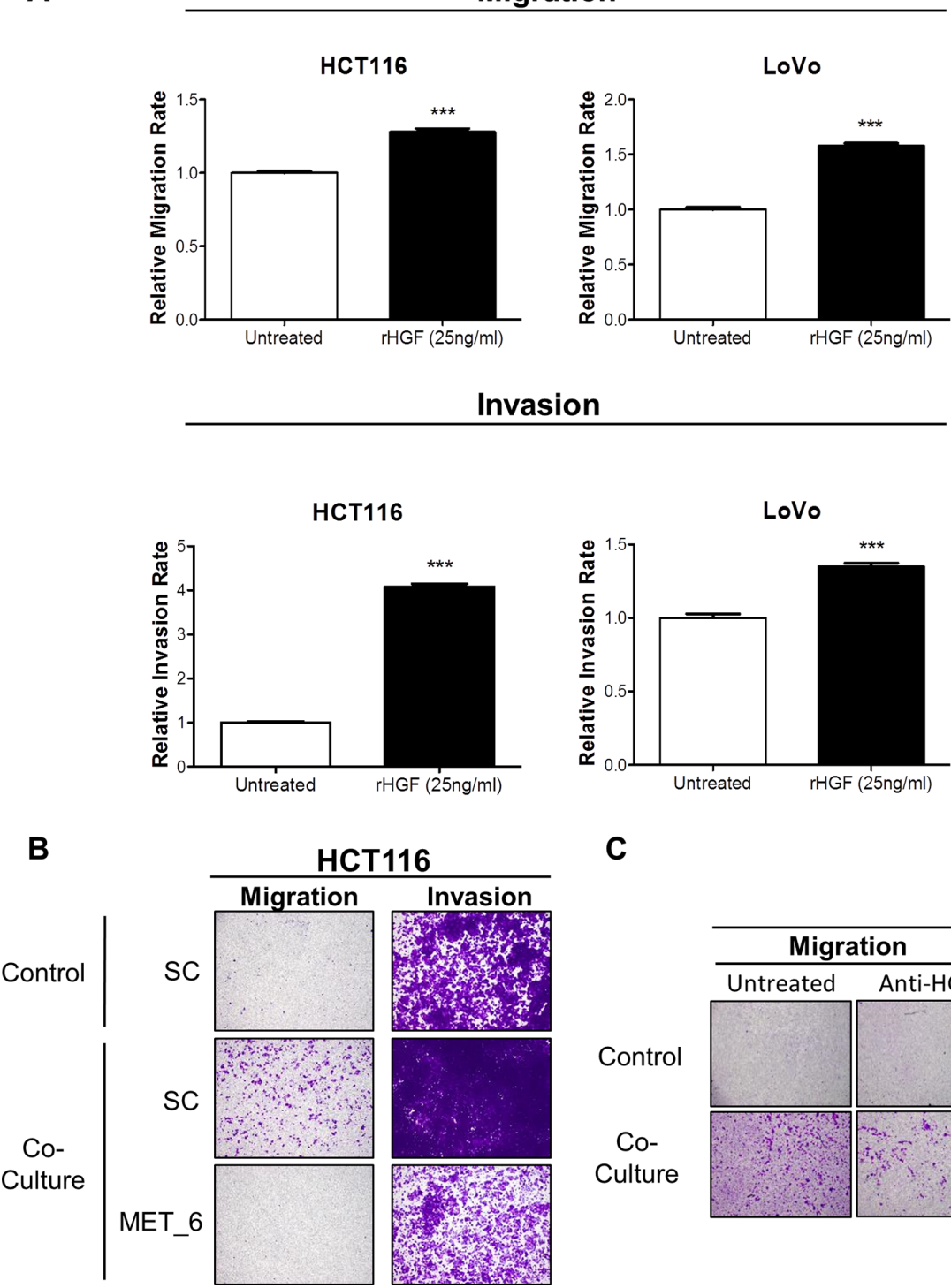

C

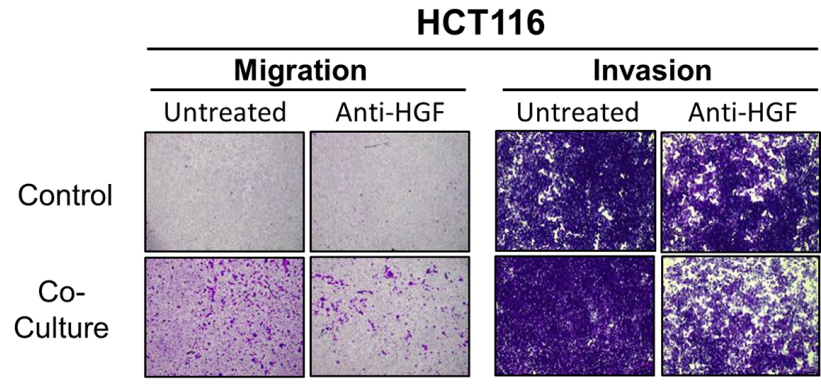

Figure 4: Recombinant HGF promotes migration and invasion of CRC cells. (A) Migration and invasion rates of HCT116 and LoVo cell lines were measured using the xCELLigence system in the absence and presence of $25 \mathrm{ng} / \mathrm{ml}$ rh-HGF. (B) Boyden chamber assessment of HCT116 migration and invasion, following transfection with $10 \mathrm{nM} \mathrm{SC}$ or $10 \mathrm{nM}$ siMET and indirect co-culture with CCD18Co cells. (C) Boyden chamber assessment of HCT116 migration and invasion following co-culture with CCD-18Co cells, in the absence or presence of anti-HGF neutralising antibody. $* * *=p<0.001$. 
3-6 molecular subtypes within stage II/III CRC [18, 19, 31-33]. Recent consensus molecular subtype analysis by the CRC Subtyping Consortium (CRCSC) has coalesced these independent classification systems into 4 Consensus Molecular Subtypes (CMS 1-4) [6]. Of these subgroups, the mesenchymal subtype (CMS4) has been associated with poor differentiation and the poorest patient outcome. Although the initial studies suggested that cancer cells of epithelial origin within CMS 4 had acquired mesenchymal traits and stem cell-like properties, resulting in increased invasion and metastatic spread, more recent studies have shown that the distinctive gene expression profiles and clinical features of CMS4 are due to their profuse stromal cell component $[7,8]$. While the latter studies argue against a widespread EMT across the entire tumor, these studies do not exclude the possibility that individual tumor cells may undergo EMT, particularly at the invasion front and in budding cells. Indeed, previous studies have shown that tumor cells at the invasive front and budding cells have a strong nuclear staining pattern for b-catenin, which is associated with loss of membranous E-cadherin and Ki-67 expression, epithelial and proliferation markers respectively $[34,35]$. Not surprisingly, high-grade tumor budding in CRC has been associated with poor patient outcome, pathological invasion, and metastasis to the lymph node and liver $[4,5]$. Based on the findings from our in vitro models, we performed focused single cell profiling of individual CRC tumor buds, in order to reveal the precise biology associated with the invasive capacity of these budding cells.

Oncogenic deregulation of the HGF/c-MET pathway has been reported in a wide range of human cancers, including breast [36], ovarian [37], CRC [25], prostate [38], pancreatic [39], gastric [40, 41] and non-small cell lung carcinoma [42], where it is often associated with drug resistance, enhanced migration/invasion, metastasis and a poor clinical outcome [41, 43, 44]. The apparent overexpression of $M E T$ at mRNA and protein levels, using qRT-PCR, microarray transcriptional profiling and IHC, has been reported by a number of independent groups in CRC [24, 26, 45-48], with a recent meta-analysis of a number of these studies offering the consensus that overexpression of c-MET and HGF occurs at a frequency of up to $\sim 78 \%$ and $\sim 95 \%$ respectively [49]. In addition, elevated c-MET expression and/or amplification has also been associated with depth of tumor (T) invasion, lymphovascular invasion, presence of regional lymph node and distant metastatic disease in CRC [24-26, 50].

In order to model CRC cell invasion, our group has previously developed invasive CRC daughter cells which

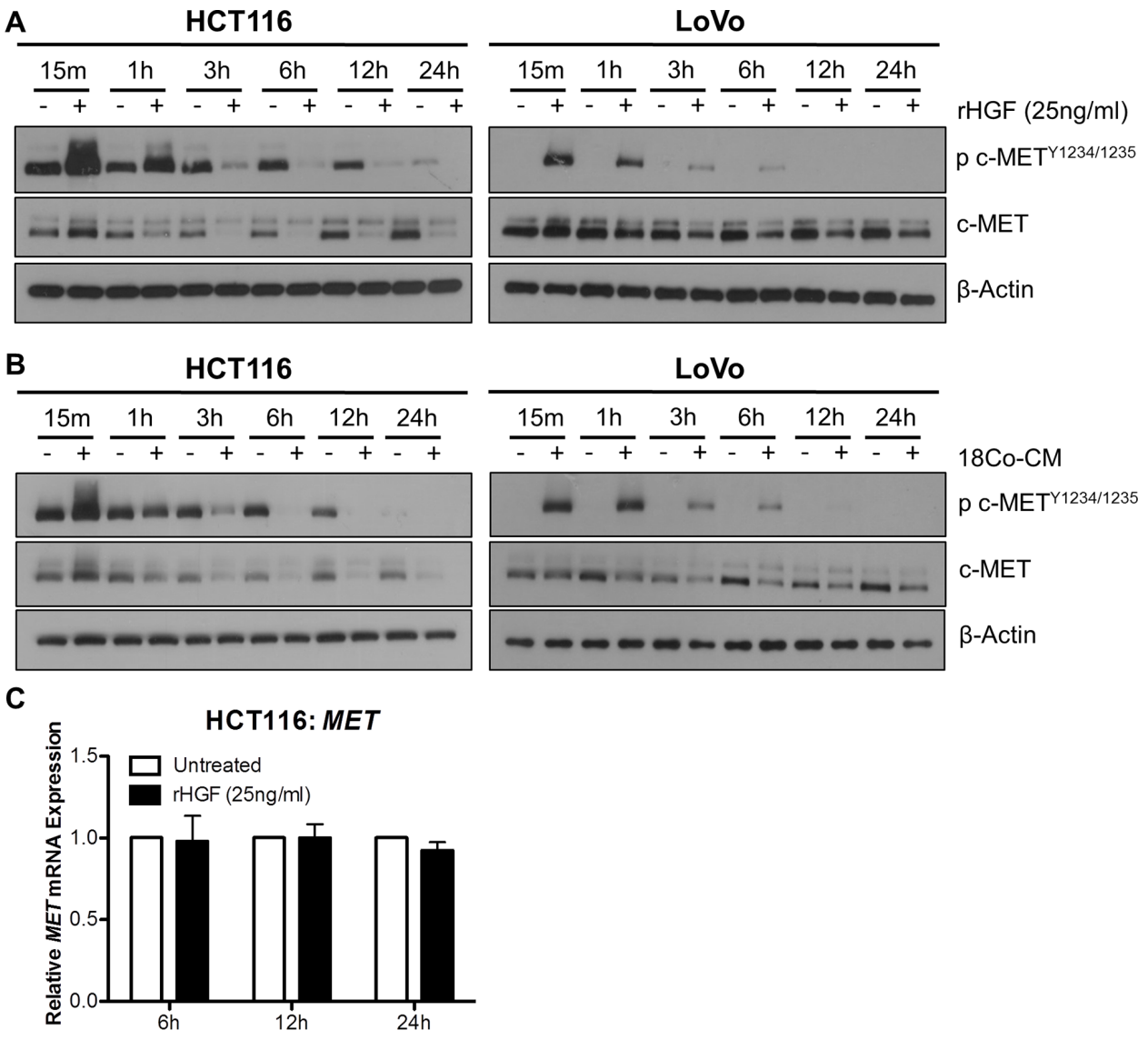

Figure 5: Exogenous HGF results in rapid downregulation of c-MET protein but not MET mRNA levels. A + B. Western blot analysis of pMET ${ }^{1234 / 1235}$ and MET levels in HCT116 and LoVo cells following stimulation with rh-HGF (A) or incubation with conditioned media from CCD-18Co cells (18Co-CM) (B) for 15 minutes, 1, 3, 6, 12 and 24 hours. (C) qRT-PCR quantification of the corresponding MET gene expression levels at 6, 12 and 24 hours following culture in the absence or presence of rh-HGF. 
displayed an EMT-like phenotype and high levels of CD44 [21]. We now show that c-MET protein and mRNA levels are highly expressed in these models and in our newly developed invasive CRC daughter cell lines and that siRNA mediated knockdown of c-MET potently inhibited migration and invasion of parental and invasive CRC cells. We next demonstrated that myofibroblast-derived conditioned medium can further potentiate migration and invasion of CRC cells through HGF-dependent activation of c-MET in CRC cells. Importantly, we found that RNAi against c-MET and monoclonal antibodies against HGF abrogated the increased migratory and/or invasion potential following co-culture of CRC cells with colon myofibroblasts. Taken together, these studies suggest a key role for HGFindependent and HGF-dependent activation of c-MET as a molecular driver of CRC cell migration/invasion in vitro.
To underpin the results of our in vitro experiments, we used a novel RNA ISH approach for single cell analysis of tumor budding foci in a cohort of stage III CRC tumor samples. These data showed marked upregulated $M E T$ mRNA expression in budding tumor cells, compared to levels obtained in tumor cells within the central tumor, regardless of the overall $M E T$ mRNA levels in the central regions. Although the CRC cases selected for this study most likely have different mutational spectra, the increased $M E T$ gene expression levels in tumor budding foci were consistently observed in every CRC tumor tissue sample analysed. This finding confirms the homogeneous nature of $M E T$ gene expression association with actively invading cells and tumor buds. Importantly, this transcriptional event was not reflected at the protein level, as measured by IHC. Previous studies, including

A

P09 07778

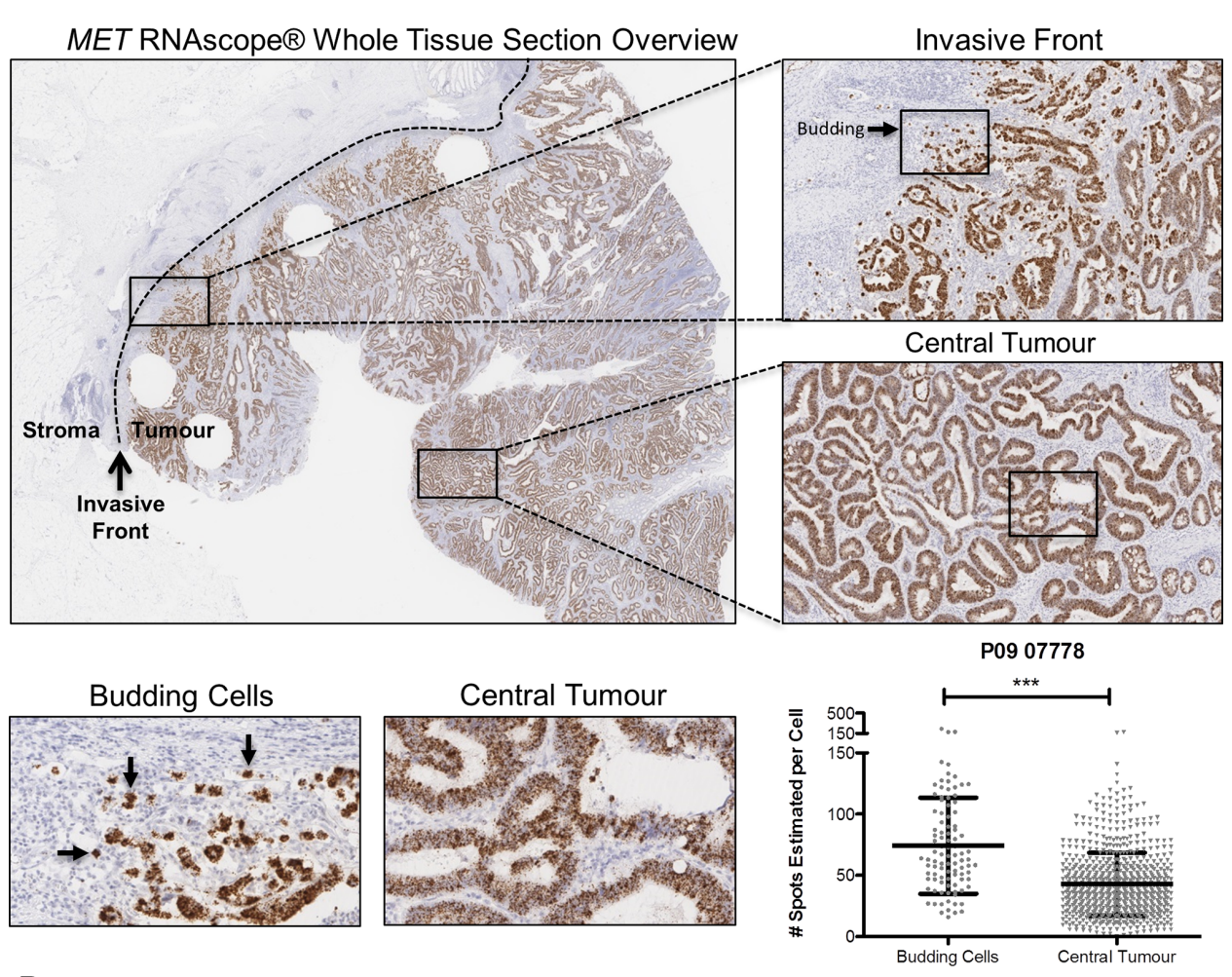

B
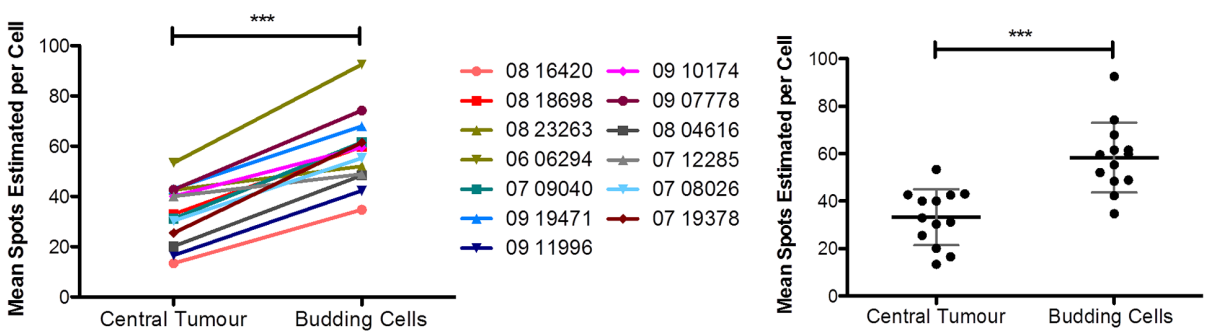

Figure 6: Budding cells at CRC invasive front harbour increased $M E T$ mRNA levels. (A) Representative image of $M E T$ RNA in situ hybridization (RNA ISH) across a full-face tumor section at low magnification (x1 top left) and higher magnification for selected regions (x5, top right). Differential expression of MET mRNA between the central tumor and budding cells at the invasive front was observed qualitatively (x20, bottom left) and determined to be significantly different using SpotStudio software analysis $(p<0.001)$ (bottom right). (B) MET mRNA levels at multiple annotated regions of interests were determined using SpotStudio software across all 13 CRC cases. Mean MET mRNA levels at each region are indicated using paired assessment (left) and group analysis (right). $* * *=p<0.001$. 
our own data, have shown that growth factor binding to plasma membrane receptor tyrosine kinases, such as the Epidermal Growth Factor Receptor (EGFR), EpHA2 and c-MET result in rapid internalization and degradation of these receptors, providing a mechanism for preventing sustained and uncontrolled pathway activation [20, 51]. A number of studies, including our current data, have shown that c-MET and HGF are detected primarily in epithelial cancer cells and stromal cells respectively [52]. These data support our hypothesis that the discordance between c-MET protein and MET gene expression, detected in tumor budding cells, is a consequence of HGF-dependent internalisation and degradation of the c-MET protein, as budding tumor cells are likely to be exposed to high physiological concentrations of stromal-derived HGF. Thus, the widespread adoption of IHC for biomarker analysis is clearly not optimal for proteins such as c-MET, which undergo rapid internalisation and degradation after exposure to stromal-derived factors.

Previous studies carried out to assess a potential prognostic role for c-MET in CRC have shown inconsistent results. These discrepant published data reflect not only the differences in technical detection methodologies used (IHC, qRT-PCR, FISH) but also the wide variety and subjective scoring criteria used for IHC [26, 53-57]. As MET gene expression is not subject to ligand-dependent negative regulation (Figure 5), our study also indicates that the use of a transcriptional semiquantitative method such as RNA ISH may be a more appropriate technology for measuring the prognostic significance of c-MET in invasive CRC. A limitation of our study was the small sample size of our pilot study, and a larger prospective study is planned to assess c-MET expression in stage II/III CRC, using both MET RNA ISH and IHC analysis of tumor budding foci, invasive front and central tumor and correlate with patient outcome. In addition, the ongoing phase I/II MErCuRIC clinical trial of combined c-MET/MEK1/2 inhibition in $R A S M T$ and $R A S W T$ metastatic CRC with aberrant c-MET expression, is assessing MET RNA ISH, IHC and DDISH in CRC liver metastasis and its potential as predictive biomarker [58].

The use of a single cell profiling method, such as RNA ISH, has allowed us to begin to uncover the transcriptional signalling that is associated with early invasive CRC. In addition, the cell-specific precision of this approach enables accurate delineation of the source of the detected transcript. RNA ISH also allows a cell-bycell dissection of the detailed biology underpinning tumor progression in $\mathrm{CRC}$, which is not routinely possible using standard transcriptomics approaches.

In conclusion, using preclinical $\mathrm{CRC}$ models and patient tissue samples, we have identified c-MET as a key regulator of CRC cell migration and invasion. A variety of therapeutic strategies have been developed to target the c-MET axis, including monoclonal antibodies and both selective and unselective tyrosine kinase inhibitors. Our data provide support for the further investigation of c-MET as a target in early stage III CRC patients who exhibit high MET mRNA levels in budding cells and the invasive front.

\section{MATERIALS AND METHODS}

\section{Cell culture}

Acquisition, authentication, and culture of HCT116, HKH-2, LoVo, and DLD-1 cells has previously been described [21]. CCD-18Co cells were purchased from American Type Tissue Culture Collection (ATCC), and cultured in Eagle's Minimum Essential Medium (EMEM) according to the manufacturers recommendations. All cell lines were authenticated (by short tandem repeat (STR)profiling and karyotyping).

\section{Selection of invasive CRC subpopulations}

BD BioCoat Matrigel Invasion Chambers (BD Biosciences) were used to isolate invasive cell subpopulations from CRC cell lines, as described previously [21]. In brief, CRC cells were seeded in serumfree Dulbecco's Modified Eagle Medium (DMEM) into the upper chamber, and allowed to invade for 72-96 hours towards chemoattractant ( $10 \%$ serum DMEM) in the lower chamber. Invaded cell populations were subsequently subcultured, and termed "invasive".

\section{In vitro migration and invasion assays}

Real-time migration and invasion were assessed in real-time using the CIM-plate 16 and the $\mathrm{xCELLigence}$ system (Roche Applied Sciences, City Country) according to the manufacturer's instructions, and as previously described [21]. For classical migration/ invasion assays, Corning Transwell PET migration Chambers (Corning) and BD BioCoat Matrigel Invasion Chambers (BD Biosciences) were used as described previously $[20,21]$.

\section{siRNA transfections, flow cytometry, western blotting, qRT-PCR, ELISA and RTK array profiling}

Transfections, Flow Cytometry, and Western blot analysis have been previously described [21]. Real-time quantitative PCR (qRT-PCR) was performed as previously described [20, 21]. The Proteome Profiler Human XL Cytokine Array Kit (R\&D Systems) was used for pRTK array profiling, and ImageJ software (Fiji) for quantification. The RayBio ${ }^{\circledR}$ Human HGF ELISA kit was used for quantification of HGF secretion, as previously described [27]. 


\section{Patients and samples for the pilot study}

For assessment of c-MET expression at the invasive edge and in the central tumor, FFPE primary CRC tumor blocks from 99 patients who received adjuvant XELOX treatment were obtained (NIB12-0034). From this cohort, H\&E stained serial whole tissue sections were microscopically examined and 13 cases were identified having i) a clear central tumor region, incorporating neoplastic glands distal from the stroma; ii) an invasive front, with a clear tumor-stroma boundary; iii) a surrounding stromal region adjacent to the invasive edge; iv) evidence of tumor budding.

\section{RNAscope staining and quantification of MET mRNA expression}

Sections from the selected 13 FFPE tumor blocks were stained for MET mRNA using the RNAscope ${ }^{\circledR} 2.0$ HD Detection Kit (Brown) for FFPE Tissues (Advanced Cell Diagnostics, Hayward, CA; \#310035). Briefly, sections were cut at $4 \mu \mathrm{m}$, air-dried overnight, baked at $60^{\circ} \mathrm{C}$ for 1 hour, dewaxed, and air-dried before pretreatments. For all probes, a standard pre-treatment protocol was used. Three RNAscope ${ }^{\circledR}$ probes were employed in this study: Homo sapiens MET (Hs-MET) (Cat. No. 423101 - sequence region 175-6505), positive control probe Homo sapiens ubiquitin C (Hs-UBC) (Cat. No. 310041 - sequence region 342-1503), and negative control probe dihydrodipicolinate reductase (bacterial dapB) (Cat. No. 310043 - sequence region 139-989). Slides were scanned using an Aperio scanner at $40 \mathrm{X}$ resolution. Quantification of $M E T$ mRNA in selected regions of interest (ROI) was performed using SpotStudio $^{\mathrm{TM}}$ software (Definiens and Advanced Cell Diagnostics). ROI's included i) budding cells at the leading edge of the tumor, defined as small clusters of five or less cells located in the stroma, which have broken away from the epithelial glands at the invasive edge, and ii) central tumor regions, defined as epithelial cells with glandular morphology located distal to the invasive edge. Results for these selected cases were expressed as mean number of spots estimated per cell.

\section{c-MET immunohistochemistry and scoring}

For IHC evaluation of c-MET protein expression, the CONFIRM Anti-Total c-MET (SP44) Rabbit Monoclonal Primary Antibody (Ventana ${ }^{\circledR}$, Cat. No. 790 4430) was used. Sections for IHC were cut at $4 \mu \mathrm{m}$ on a rotary microtome, dried at $37^{\circ} \mathrm{C}$ overnight, and then used for IHC, which was performed on an automated immunostainer (Leica Bond-Max, Milton Keynes, UK). A validated and optimized in-house protocol was used for c-MET IHC. Antigen-binding sites were detected with a polymer-based detection system (Bond, Newcastle Upon Tyne, UK; cat. no. DS9800). All sections were visualized with diaminobenzidine, counterstained with hematoxylin, and mounted in DPX.

Staining reaction for c-MET IHC was graded on a 4 four-tiered system: 0 : no staining or $<10 \%$ of tumor cells stained at weak intensity, 1+: weak staining in $>$ $10 \%$ of the tumor cells in the region of interest (ROI), $2+$ : moderate staining in $>10 \%$ of the tumor cells in the ROI, $3+$ : strong staining in $>10 \%$ of the tumor cells in the ROI. Only cytoplasmic and membranous expression was evaluated.

\section{Gene expression dataset}

Gene expression profiles from an independent CRC dataset were downloaded from NCBI Gene Expression Omnibus (GEO) (http://www.ncbi.nlm.nih.gov/geo/) under accession number GSE39396. GSE39396 contains microarray profiles from fresh colorectal specimens where Fluorescence Activated Cell Sorting (FACS) selected cells into specific endothelial $[\mathrm{CD} 45(+), \operatorname{EPCAM}(-)$, CD31(-), FAP(-)], epithelial [CD45(-) $\operatorname{EPCAM}(+)$, CD31(-), FAP(-)], leukocyte [CD45(-), $\operatorname{EPCAM}(-)$, $\mathrm{CD} 31(+), \mathrm{FAP}(-)]$ and fibroblast $[\mathrm{CD} 45(-), \operatorname{EPCAM}(-)$, CD31(-), FAP $(+)$ ] populations.

\section{Transcriptional analysis}

Partek Genomics Suite ${ }^{\circledR} 6.6$ software (Partek Inc.) was used for dataset analysis. The data was uploaded and underwent RMA normalisation prior to downstream analysis. Expression values for the 4 probesets representing $M E T$ were selected (203510_PM_at; 211599 PM_x_at; 213807_PM_x_at; 213816_PM_s_at). For the purpose of clustering, the data matrices were standardized to the median value of probeset expression. Following standardization, 2-dimensional hierarchical clustering was performed (samples x probe sets/genes). Hierarchical clustering was carried out using Euclidean distance with Ward's linkage method.

\section{Statistical analyses}

GraphPad Prism 5 Software (Graphpad.com) was used for all statistical tests of in vitro data. Relative expression box and whisker data was plotted as median probeset values $(10 \%$ and $90 \%$ values indicated by whiskers). Additionally, relative qRT-PCR expression, cell cycle values and migration/invasion rates were also plotted. Unpaired Students $t$-tests were used to determine significance $(p<0.05)$ of indicated groups. ANOVA tests were used to determine statistical significance of multiple groups, with Tukey's post hoc correction. 


\section{ACKNOWLEDGMENTS}

This work was undertaken with the assistance of the Northern Ireland Molecular Pathology Laboratory (NIMPL) and the Northern Ireland BioBank (NIB) based at Queens University Belfast (QUB). We would like to thank Manuel Salto-Tellez (MST) for H\&E assessment of CRC tissues sections.

\section{CONFLICTS OF INTEREST}

CAB: None; PDD: None; VB: None; SMCQ: None; HK: None; SC: None; JJ: None; WLM: None; DGM: None; ML: None; SD: None; PGJ: Previous Founder and Shareholder of Almac Diagnostics; CV6 Therapeutics: Expert Advisor and Shareholder; Chugai Pharmaceuticals: Consultant; SVS: None.

\section{GRANT SUPPORT}

Funding was supported by Cancer Research UK (C212/A13721); Cancer Research UK fellowship (C13749/A13172) and by MErCuRIC, funded by the European Commission's Framework Programme 7, under contract \#602901.

\section{REFERENCES}

1. Haggar FA, Boushey RP. Colorectal cancer epidemiology: incidence, mortality, survival, and risk factors. Clin Colon Rectal Surg. 2009; 22:191-197.

2. Mitrovic B, Schaeffer DF, Riddell RH, Kirsch R. Tumor budding in colorectal carcinoma: time to take notice. Mod Pathol. 2012; 25:1315-1325.

3. Jass JR, Love SB, Northover JM. A new prognostic classification of rectal cancer. Lancet. 1987; 1:1303-1306.

4. Wang LM, Kevans D, Mulcahy H, O'Sullivan J, Fennelly D, Hyland J, O'Donoghue D, Sheahan K. Tumor budding is a strong and reproducible prognostic marker in T3N0 colorectal cancer. Am J Surg Pathol. 2009; 33:134-141.

5. Syk E, Lenander C, Nilsson PJ, Rubio CA, Glimelius B. Tumour budding correlates with local recurrence of rectal cancer. Colorectal Dis. 2011; 13:255-262.

6. Guinney J, Dienstmann R, Wang X, de Reynies A, Schlicker A, Soneson C, Marisa L, Roepman P, Nyamundanda G, Angelino P, Bot BM, Morris JS, Simon IM, et al. The consensus molecular subtypes of colorectal cancer. Nat Med. 2015; 21:1350-1356.

7. Calon A, Lonardo E, Berenguer-Llergo A, Espinet E, Hernando-Momblona X, Iglesias M, Sevillano M, PalomoPonce S, Tauriello DV, Byrom D, Cortina C, Morral C, Barcelo C, et al. Stromal gene expression defines poorprognosis subtypes in colorectal cancer. Nat Genet. 2015; 47:320-329.

8. Isella C, Terrasi A, Bellomo SE, Petti C, Galatola G, Muratore A, Mellano A, Senetta R, Cassenti A, Sonetto C,
Inghirami G, Trusolino L, Fekete Z, et al. Stromal contribution to the colorectal cancer transcriptome. Nat Genet. 2015; 47:312-319.

9. Jung A, Schrauder M, Oswald U, Knoll C, Sellberg P, Palmqvist R, Niedobitek G, Brabletz T, Kirchner T. The invasion front of human colorectal adenocarcinomas shows co-localization of nuclear beta-catenin, cyclin D1, and $\mathrm{p} 16 \mathrm{INK} 4 \mathrm{~A}$ and is a region of low proliferation. Am J Pathol. 2001; 159:1613-1617.

10. Vermeulen L, De Sousa EMF, van der Heijden M, Cameron K, de Jong JH, Borovski T, Tuynman JB, Todaro M, Merz C, Rodermond H, Sprick MR, Kemper K, Richel DJ, et al. Wnt activity defines colon cancer stem cells and is regulated by the microenvironment. Nat Cell Biol. 2010; 12:468-476.

11. Fodde R, Brabletz T. Wnt/beta-catenin signaling in cancer stemness and malignant behavior. Curr Opin Cell Biol. 2007; 19:150-158.

12. Brabletz T, Jung A, Spaderna S, Hlubek F, Kirchner T. Opinion: migrating cancer stem cells - an integrated concept of malignant tumour progression. Nat Rev Cancer. 2005; 5:744-749.

13. De Wever O, Mareel M. Role of tissue stroma in cancer cell invasion. J Pathol. 2003; 200:429-447.

14. Joyce JA, Pollard JW. Microenvironmental regulation of metastasis. Nat Rev Cancer. 2009; 9:239-252.

15. Wikberg ML, Edin S, Lundberg IV, Van Guelpen B, Dahlin AM, Rutegard J, Stenling R, Oberg A, Palmqvist R. High intratumoral expression of fibroblast activation protein (FAP) in colon cancer is associated with poorer patient prognosis. Tumour Biol. 2013; 34:1013-1020.

16. Berdiel-Acer M, Berenguer A, Sanz-Pamplona R, Cuadras D, Sanjuan X, Paules MJ, Santos C, Salazar R, Moreno V, Capella G, Villanueva A, Mollevi DG. A 5-gene classifier from the carcinoma-associated fibroblast transcriptomic profile and clinical outcome in colorectal cancer. Oncotarget. 2014; 5:6437-6452. doi: 10.18632/oncotarget.2237.

17. Wilson TR, Fridlyand J, Yan Y, Penuel E, Burton L, Chan E, Peng J, Lin E, Wang Y, Sosman J, Ribas A, Li J, Moffat J, et al. Widespread potential for growth-factor-driven resistance to anticancer kinase inhibitors. Nature. 2012; 487:505-509.

18. Sadanandam A, Lyssiotis CA, Homicsko K, Collisson EA, Gibb WJ, Wullschleger S, Ostos LC, Lannon WA, Grotzinger C, Del Rio M, Lhermitte B, Olshen AB, Wiedenmann B, et al. A colorectal cancer classification system that associates cellular phenotype and responses to therapy. Nat Med. 2013; 19:619-625.

19. Marisa L, de Reynies A, Duval A, Selves J, Gaub MP, Vescovo L, Etienne-Grimaldi MC, Schiappa R, Guenot D, Ayadi M, Kirzin S, Chazal M, Flejou JF, et al. Gene expression classification of colon cancer into molecular subtypes: characterization, validation, and prognostic value. PLoS Med. 2013; 10:e1001453.

20. Dunne PD, Dasgupta S, Blayney JK, McArt DG, Redmond KL, Weir JA, Bradley CA, Sasazuki T, Shirasawa S, Wang T, Srivastava S, Ong CW, Arthur K, et al. EphA2 Expression Is a Key Driver of Migration and 
Invasion and a Poor Prognostic Marker in Colorectal Cancer. Clin Cancer Res. 2016; 22:230-242.

21. Dunne PD, McArt DG, Blayney JK, Kalimutho M, Greer S, Wang T, Srivastava S, Ong CW, Arthur K, Loughrey M, Redmond K, Longley DB, Salto-Tellez M, et al. AXL is a key regulator of inherent and chemotherapy-induced invasion and predicts a poor clinical outcome in early-stage colon cancer. Clin Cancer Res. 2014; 20:164-175.

22. Boccaccio C, Comoglio PM. Invasive growth: a MET-driven genetic programme for cancer and stem cells. Nat Rev Cancer. 2006; 6:637-645.

23. Furlan A, Kherrouche Z, Montagne R, Copin MC, Tulasne D. Thirty years of research on met receptor to move a biomarker from bench to bedside. Cancer Res. 2014; 74:6737-6744.

24. Takeuchi H, Bilchik A, Saha S, Turner R, Wiese D, Tanaka M, Kuo C, Wang HJ, Hoon DS. c-MET expression level in primary colon cancer: a predictor of tumor invasion and lymph node metastases. Clin Cancer Res. 2003; 9: 1480-1488.

25. Di Renzo MF, Olivero M, Giacomini A, Porte H, Chastre E, Mirossay L, Nordlinger B, Bretti S, Bottardi S, Giordano S, et al. Overexpression and amplification of the met/HGF receptor gene during the progression of colorectal cancer. Clin Cancer Res. 1995; 1:147-154.

26. Zeng ZS, Weiser MR, Kuntz E, Chen CT, Khan SA, Forslund A, Nash GM, Gimbel M, Yamaguchi Y, Culliford ATt, D'Alessio M, Barany F, Paty PB. c-Met gene amplification is associated with advanced stage colorectal cancer and liver metastases. Cancer Lett. 2008; 265:258-269.

27. Carson R, Celtikci B, Fenning C, Javadi A, Crawford N, Perez-Carbonell L, Lawler M, Longley DB, Johnston PG, Van Schaeybroeck S. HDAC Inhibition Overcomes Acute Resistance to MEK Inhibition in BRAF-Mutant Colorectal Cancer by Downregulation of c-FLIPL. Clin Cancer Res. $2015 ; 21: 3230-3240$.

28. Van Schaeybroeck S, Kalimutho M, Dunne PD, Carson R, Allen W, Jithesh PV, Redmond KL, Sasazuki T, Shirasawa S, Blayney J, Michieli P, Fenning C, Lenz HJ, et al. ADAM17dependent c-MET-STAT3 signaling mediates resistance to MEK inhibitors in KRAS mutant colorectal cancer. Cell Rep. 2014; 7:1940-1955.

29. Brabletz T. To differentiate or not--routes towards metastasis. Nat Rev Cancer. 2012; 12:425-436.

30. Del Pozo Martin Y, Park D, Ramachandran A, Ombrato L, Calvo F, Chakravarty P, Spencer-Dene B, Derzsi S, Hill CS, Sahai E, Malanchi I. Mesenchymal Cancer Cell-Stroma Crosstalk Promotes Niche Activation, Epithelial Reversion, and Metastatic Colonization. Cell Rep. 2015; 13:2456-2469.

31. De Sousa EMF, Wang X, Jansen M, Fessler E, Trinh A, de Rooij LP, de Jong JH, de Boer OJ, van Leersum R, Bijlsma MF, Rodermond $\mathrm{H}$, van der Heijden $\mathrm{M}$, van Noesel CJ, et al. Poor-prognosis colon cancer is defined by a molecularly distinct subtype and develops from serrated precursor lesions. Nat Med. 2013; 19:614-618.

32. Roepman P, Schlicker A, Tabernero J, Majewski I, Tian S, Moreno V, Snel MH, Chresta CM, Rosenberg R, Nitsche U,
Macarulla T, Capella G, Salazar R, et al. Colorectal cancer intrinsic subtypes predict chemotherapy benefit, deficient mismatch repair and epithelial-to-mesenchymal transition. Int J Cancer. 2014; 134:552-562.

33. Cancer Genome Atlas N. Comprehensive molecular characterization of human colon and rectal cancer. Nature. 2012; 487:330-337.

34. Brabletz T, Jung A, Reu S, Porzner M, Hlubek F, KunzSchughart LA, Knuechel R, Kirchner T. Variable beta-catenin expression in colorectal cancers indicates tumor progression driven by the tumor environment. Proc Natl Acad Sci U S A. 2001; 98:10356-10361.

35. Brabletz S, Schmalhofer O, Brabletz T. Gastrointestinal stem cells in development and cancer. J Pathol. 2009; 217: 307-317.

36. Garcia S, Dales JP, Charafe-Jauffret E, Carpentier-Meunier S, Andrac-Meyer L, Jacquemier J, Andonian C, Lavaut MN, Allasia C, Bonnier P, Charpin C. Poor prognosis in breast carcinomas correlates with increased expression of targetable CD146 and c-Met and with proteomic basal-like phenotype. Hum Pathol. 2007; 38:830-841.

37. Wong AS, Pelech SL, Woo MM, Yim G, Rosen B, Ehlen T, Leung PC, Auersperg N. Coexpression of hepatocyte growth factor-Met: an early step in ovarian carcinogenesis? Oncogene. 2001; 20:1318-1328.

38. Humphrey PA, Zhu X, Zarnegar R, Swanson PE, Ratliff TL, Vollmer RT, Day ML. Hepatocyte growth factor and its receptor (c-MET) in prostatic carcinoma. Am J Pathol. 1995; 147:386-396.

39. Di Renzo MF, Poulsom R, Olivero M, Comoglio PM, Lemoine NR. Expression of the Met/hepatocyte growth factor receptor in human pancreatic cancer. Cancer Res. 1995; 55:1129-1138.

40. Soman NR, Correa P, Ruiz BA, Wogan GN. The TPR-MET oncogenic rearrangement is present and expressed in human gastric carcinoma and precursor lesions. Proc Natl Acad Sci U S A. 1991; 88:4892-4896.

41. Kuniyasu H, Yasui W, Yokozaki H, Kitadai Y, Tahara E. Aberrant expression of c-met mRNA in human gastric carcinomas. Int J Cancer. 1993; 55:72-75.

42. Ichimura E, Maeshima A, Nakajima T, Nakamura T. Expression of c-met/HGF receptor in human non-small cell lung carcinomas in vitro and in vivo and its prognostic significance. Jpn J Cancer Res. 1996; 87:1063-1069.

43. Ghoussoub RA, Dillon DA, D'Aquila T, Rimm EB, Fearon ER, Rimm DL. Expression of c-met is a strong independent prognostic factor in breast carcinoma. Cancer. 1998; 82:1513-1520.

44. Ueki T, Fujimoto J, Suzuki T, Yamamoto H, Okamoto E. Expression of hepatocyte growth factor and its receptor c-met proto-oncogene in hepatocellular carcinoma. Hepatology. 1997; 25:862-866.

45. Seiden-Long IM, Brown KR, Shih W, Wigle DA, Radulovich N, Jurisica I, Tsao MS. Transcriptional targets of hepatocyte growth factor signaling and Ki-ras oncogene activation in colorectal cancer. Oncogene. 2006; 25:91-102. 
46. Kammula US, Kuntz EJ, Francone TD, Zeng Z, Shia J, Landmann RG, Paty PB, Weiser MR. Molecular coexpression of the c-Met oncogene and hepatocyte growth factor in primary colon cancer predicts tumor stage and clinical outcome. Cancer Lett. 2007; 248:219-228.

47. Ma PC, Tretiakova MS, MacKinnon AC, Ramnath N, Johnson C, Dietrich S, Seiwert T, Christensen JG, Jagadeeswaran R, Krausz T, Vokes EE, Husain AN, Salgia R. Expression and mutational analysis of MET in human solid cancers. Genes Chromosomes Cancer. 2008; 47:1025-1037.

48. Umeki K, Shiota G, Kawasaki H. Clinical significance of c-met oncogene alterations in human colorectal cancer. Oncology. 1999; 56:314-321.

49. Sierra JR, Tsao MS. c-MET as a potential therapeutic target and biomarker in cancer. Ther Adv Med Oncol. 2011; 3: S21-35.

50. Zeng Z, Weiser MR, D'Alessio M, Grace A, Shia J, Paty PB. Immunoblot analysis of c-Met expression in human colorectal cancer: overexpression is associated with advanced stage cancer. Clin Exp Metastasis. 2004; 21: 409-417.

51. Di Fiore PP, Gill GN. Endocytosis and mitogenic signaling. Curr Opin Cell Biol. 1999; 11:483-488.

52. Otte JM, Schmitz F, Kiehne K, Stechele HU, Banasiewicz T, Krokowicz P, Nakamura T, Folsch UR, Herzig K. Functional expression of $\mathrm{HGF}$ and its receptor in human colorectal cancer. Digestion. 2000; 61:237-246.

53. De Oliveira AT, Matos D, Logullo AF, SR DAS, Neto RA, Filho AL, Saad SS. MET Is highly expressed in advanced stages of colorectal cancer and indicates worse prognosis and mortality. Anticancer Res. 2009; 29:4807-4811.

54. Resnick MB, Routhier J, Konkin T, Sabo E, Pricolo VE. Epidermal growth factor receptor, c-MET, beta-catenin, and p53 expression as prognostic indicators in stage II colon cancer: a tissue microarray study. Clin Cancer Res. 2004; 10:3069-3075.

55. Al-Maghrabi J, Emam E, Gomaa W, Saggaf M, Buhmeida A, Al-Qahtani M, Al-Ahwal M. c-MET immunostaining in colorectal carcinoma is associated with local disease recurrence. BMC Cancer. 2015; 15:676.

56. Voutsina A, Tzardi M, Kalikaki A, Zafeiriou Z, Papadimitraki E, Papadakis M, Mavroudis D, Georgoulias V. Combined analysis of KRAS and PIK3CA mutations, MET and PTEN expression in primary tumors and corresponding metastases in colorectal cancer. Mod Pathol. 2013; 26: 302-313.

57. Liu Y, Yu XF, Zou J, Luo ZH. Prognostic value of c-Met in colorectal cancer: a meta-analysis. World J Gastroenterol. 2015; 21:3706-3710.

58. Van Schaeybroeck S, Rolfo CD, Elez E, Kelly S, Houlden J, Collins L, Love L, Andre T, Lawler M, di Nicolantonio F, Grayson M, Popovici V, Bardelli A, et al. MErCuRIC1: A Phase I study of MEK1/2 inhibitor PD-0325901 with cMET inhibitor crizotinib in RASMT and RASWT (with aberrant c-MET) metastatic colorectal cancer (mCRC) patients. J Clin Oncol 33, 2015 (suppl; abstr TPS3632). 2015. 